\title{
BMJ Open Interventions for improving treatment outcomes in adolescents on antiretroviral therapy with unsuppressed viral loads: a systematic review protocol
}

\author{
Farai Kevin Munyayi (D) , ${ }^{1,2}$ Brian van Wyk ${ }^{3}$
}

To cite: Munyayi FK, van Wyk B. Interventions for improving treatment outcomes in adolescents on antiretroviral therapy with unsuppressed viral loads: a systematic review protocol. BMJ Open 2021;11:e049452. doi:10.1136/ bmjopen-2021-049452

- Prepublication history and additional supplemental material for this paper are available online. To view these files, please visit the journal online (http://dx.doi.org/10.1136/ bmjopen-2021-049452).

Received 24 January 2021 Accepted 12 August 2021

Check for updates

(C) Author(s) (or their employer(s)) 2021. Re-use permitted under CC BY-NC. No commercial re-use. See rights and permissions. Published by BMJ.

${ }^{1}$ School of Public Health, University of the Western Cape, Bellville, South Africa

${ }^{2}$ Clinical services, I-TECH Namibia, Windhoek, Namibia ${ }^{3}$ School of Public health, University of the Western Cape Faculty of Community and Health Sciences, Cape Town, South Africa

Correspondence to

Farai Kevin Munyayi fmfanchom@gmail.com

\section{ABSTRACT}

Introduction Adolescents represent one of the most underserved population groups among people living with HIV. With successes in the elimination of mother to child transmission initiatives and advances in paediatric HIV treatment programmes, a large population of HIV-infected children are surviving into adolescence. Adolescence presents unique challenges that increase the risk of non-suppressed viral loads in adolescents living with HIV (ALHIV). There is a need to develop, implement and test interventions to improve viral suppression among ALHIV. Systematic reviews of recent studies present scarce and inconclusive evidence of effectiveness of current interventions, especially for adolescents. This protocol provides a description of a planned review of interventions to improve treatment outcomes among unsuppressed ALHIV.

Methods and analysis A comprehensive search string will be used to search six bibliographic databases: PubMed/MEDLINE, Sabinet, EBSCOhost, CINAHL, Scopus and ScienceDirect, for relevant studies published between 2010 and 2020 globally, and grey literature. Identified articles will be exported into Mendeley Reference Management software and two independent reviewers will screen the titles, abstracts and full texts for eligibility. A third reviewer will resolve any discrepancies between the two initial reviewers. Studies reporting on interventions to improve viral suppression, retention and adherence for adolescents will be considered for inclusion. The systematic review will be performed and reported according to the Preferred Reporting Items for Systematic Reviews and Meta-Analyses Protocols. Where feasible, a meta-analysis will be conducted using Stata Statistical Software: Release V.16. The quality of the studies and risk of bias will be assessed using the Critical Appraisal Skills Programme checklists and Risk of Bias in Non-randomised Studies of Interventions tool, respectively.

Ethics and dissemination The systematic review entails abstracting and reviewing already publicly available data rather than any involvement of participants, therefore, no ethical clearance will be required. Results will be shared with relevant policy-makers, programme managers and service providers, and published and share through conferences and webinars.

PROSPERO registration number CRD42021232440.
Strengths and limitations of this study

- This review contributes to the available knowledge on evaluated interventions targeting adolescents with unsuppressed viral loads that could inform adolescent-friendly HIV services guidelines in both health facilities and community settings.

- This protocol uses the standardised Preferred Reporting Items for Systematic Reviews and MetaAnalyses Protocols 2015 guidelines.

- The search strategy uses six major electronic databases which will include peer-reviewed articles and grey literature.

- A potential limitation of this study includes the limitation to studies published in English language only, which has the potential to miss other relevant studies published in other languages.

- The study limitations may be associated with the timeline for the search strategy (2010-2020).

\section{INTRODUCTION}

Along with the successes in elimination of mother-to-child transmission initiatives worldwide and developments in paediatric HIV treatment, more children with HIV are surviving to reach adolescence. ${ }^{1}$ Innovations for early infant diagnosis, such as point-of-care diagnostics and prompt initiation of life-saving HIV treatment, have meant a large population of perinatally HIVinfected children are growing into adolescence. $^{2}$ A mixed adolescent population exists consisting of perinatally infected and behaviourally infected adolescents living with HIV (ALHIV) who may respond to interventions differently. ${ }^{3}$ Worldwide, 1.6 million adolescents aged 10-19 years were estimated to be living with HIV in 2018; with approximately $85 \%$ residing in sub-Saharan Africa. ${ }^{45}$ WHO defines adolescents as individuals aged between 10 and 19 years. $^{6}$ The proposed systematic review seeks to look at literature on both perinatally and behaviourally infected adolescents, aged 10-19 years. Although data 
on HIV treatment among adolescents are scarce, access and uptake of comprehensive care and treatment packages among this vulnerable population group is reportedly lower than adults. ${ }^{7}$ In 2019, only 53\% (36\%-64\%) of children aged $0-14$ years were accessing treatment as compared with $68 \%(54 \%-80 \%)$ coverage among 15 years and older individuals living with HIV. ${ }^{8}$ In the majority of sub-Saharan Africa countries, public sector health settings are ill equipped to provide guidance and adequate support for ALHIV to stay engaged in HIV care and adhere to treatment regimens. ${ }^{9}$

Good adherence to treatment regimens and consistent engagement in HIV care is essential for achieving sustained viral suppression, which is vital for ensuring the well-being of ALHIV and reducing chances of onward HIV transmission. ${ }^{10}$ Poor adherence to antiretrovirals increases the risk of emergence of drug resistance mutations, reduces treatment efficacy, thereby advancing disease progression and reducing future treatment options and increasing the risk of further transmission driven by unsuppressed viral loads. ${ }^{11}$ Despite reportedly high levels of adherence to antiretroviral therapy (ART) globally $(>95 \%)$, diminishing adherence levels over time remains a concern, primarily the loss of patients during scaling up of HIV programmes. ${ }^{12}$ Evidence-based strategies to mitigate adherence challenges for individuals on HIV treatment include facility and community-based interventions, which includes individual or group adherence counselling, pharmacist counselling and medication fast-tracking, mHealth, home-based/community-based strategies, financial incentives, nutritional support, motivational interviewing and awarding of disability grants. ${ }^{13}$ Individual counselling, peer-led support groups and different models of group adherence counselling interventions, such as teen clubs, are the most common interventions for ALHIV.

Adolescents receiving HIV treatment have distinct and unique needs and are notably underserved worldwide in national HIV responses. Ultimately, this negatively affects access to ART for adolescents, and results in suboptimal ART adherence and poorer treatment outcomes including achieving sustained viral suppression. ${ }^{14}$ WHO defines viral suppression as a maintained viral load count of less than 1000 RNA copies/mL of plasma. ${ }^{15}$ However, recent debates have suggested reducing the suppression threshold to 200 copies/mL, informed by evidence from HPTN 052 findings, PARTNERS and Opposites Attract, who all reported that successful ART with viral suppression prevented transmission when viral load was undetectable below 200 copies $/ \mathrm{mL} .{ }^{16}$ Variations in viral load assay limit of detection has also been tied to the debates around threshold setting for viral load suppression, and these need to be paid attention to in any evaluation.

A systematic review conducted in 2014 estimated global ART adherence levels ranging between $16 \%$ and $99 \%$ among adolescents and a meta-analysis of data representing adolescents and young adults between 12 and 24 years found a pooled adherence estimate of $84 \%$ in
53 Asian and African countries. Viral suppression (cutoff $<400$ copies $/ \mathrm{mL}$ ) rates were between $52 \%$ and $87 \%$ in Asia, $27 \%$ and $89 \%$ in Africa, and between $37.5 \%$ and $49 \%$ in Central and South America. ${ }^{17}$ A 2018 systematic review of 11 studies on factors impacting ART adherence among HIV positive adolescents in sub-Saharan Africa reported a complex web of 29 facilitators and 44 barriers among adolescents. These factors include stigma, forgetfulness, ART side effects and lack of assistance as main barriers, while knowledge of status, caregiver support and peer support groups were the dominant facilitators. ${ }^{18}$ Other barriers and facilitators of treatment adherence among adolescents in countries in the global south have been noted as family support, a heavy pill burden/treatment regimen, attitudes towards medication and various health delivery system factors. ${ }^{19}$ Often these studies reported on adherence only using self-report or pill counts, with no viral load results reported. Self-reported adherence is mostly challenging, especially in adolescents, as clearly demonstrated in Pre-Exposure Prophylaxis (PrEP) studies where there was no correlation between therapeutic drug monitoring and self-report especially in younger populations. ${ }^{20}$

Despite recent increased focus and several systematic reviews synthesising interventions to improve treatment outcomes among adolescents, we could not find reviews specifically focusing on studies targeting unsuppressed adolescents. Although our focus is predominantly on viral suppression, broader outcomes such as adherence and retention in care will be considered. Previous reviews have identified specific types of interventions that show promise for improved adherence to ART and retention in HIV care with very limited quantity of studies and low quality in several studies focusing on adolescents aged 10-19 years published up until $2015 .{ }^{21}$ A review by MacPherson et al focused on studies published between 2001 and 2014 evaluating effectiveness of interventions on service delivery to improve linkage to treatment initiation, retention in HIV care and ART adherence among ALHIV aged 10-19 years. ${ }^{14}$ Considering that only 11 studies were included in the study, with low-to-moderate methodological quality, from high-income countries and none from countries with a generalised HIV epidemic, the authors suggested further investigations on their findings which included promising results from individual and group education and counselling, increased accessibility to clinics, youth-friendly services and financial incentives. $^{14}$

Casale et al reviewed studies published between January 2016 and June 2018, and provided an update on evidence from empirical evaluations on interventions designed to enhance ART adherence and retention in care for adolescents aged 10-19 years and youth aged 15-24 years living with HIV. ${ }^{21}$ The authors reported on 10 relevant studies, mostly from sub-Saharan Africa, using six clinic-level interventions, three community-level or household-level intervention, and one mHealth trial at individual level, and they concluded that there is a need for continued 
development and testing of multifaceted interventions over and above facility-based interventions, that address broader socioeconomic barriers, prioritising adolescents aged 10-19 years, if we are to address the treatment gap among this population group. ${ }^{21}$ A more recent review of studies published from September 2015 to January 2019 by Lindsey et al reported that ART adherence interventions for adolescents and youth implemented in low- and middle-income countries showed mixed results from interventions at patient-level and health services level, with none of the interventions improving treatment adherence or virologic suppression. ${ }^{22}$ The authors argued that intensive home-based case management approaches to care hold much promise in improving treatment outcomes in adolescents and youth and warrant further research. ${ }^{22}$ Most recent published studies focusing on interventions such as Enhanced Adherence Counselling and Community Adolescent Treatment Supporters to improve treatment outcomes among ALHIV challenged with viral suppression and retention in care may provide new evidence for consideration. ${ }^{23-25}$

\section{RATIONALE FOR THE REVIEW}

Globally, it is apparent that there has been an increased focus in addressing the treatment gap among ALHIV, and in recent years, there have been a number of systematic reviews focusing on interventions to improve ART adherence and retention in HIV care among adolescents and young adults in general. While the gaps described above and the systematic reviews cited present opportunities and specific interventions that show promise, they also highlight the limited quantity and low-to-moderate quality of studies and evidence on interventions targeting ALHIV. These reviews commonly include interventions for all adolescents and youth, despite their viral load suppression levels, which include clinic-level interventions, individual-level and community-level or household-level interventions. Considering the urgent need to design, implement and test interventions to keep adolescents in care and achieve and maintain viral load suppression, we propose to review studies that specifically evaluated interventions targeting adolescents challenged with staying engaged in care and maintaining viral suppression. This review will focus on adolescents experiencing poor treatment outcomes to provide an updated data synthesis of all adherence and retention interventions to close the treatment gap. We aim to broaden the synthesised knowledge base to include new and innovative interventions for this particular population, at health facility, community and household levels, and potentially reinforce or modify already existing promising interventions for achievement of sustained viral suppression.

\section{METHODS}

This systematic review will use the seven steps as set out by Eggar and Smith. ${ }^{26}$ The seven steps are outlined as
(1) formulate the review question; (2) define the inclusion and exclusion criteria; (3) develop a search strategy; (4) study selection; (5) assess the quality of studies; (6) extract data and (7) analyse or synthesise the data.

\section{REVIEW QUESTION}

The following questions will guide the review of the literature published between 2010 and 2020:

1. What interventions are being implemented to improve treatment outcomes for adolescents on ART with challenges suppressing their viral load?

2. How effective have the interventions been in achieving better treatment outcomes among adolescents on ART with challenges suppressing their viral load?

\section{INCLUSION AND EXCLUSION CRITERIA}

The literature search will be guided by the following inclusion criteria:

1. Published studies in peer-reviewed journals and grey literature.

2. The study population includes ALHIV aged 10-19 years, both perinatally and behaviourally infected adolescents included.

3. Reported the association between the intervention, and viral suppression, retention and adherence.

4. Reported comparisons in quantitative measures of viral suppression, retention and adherence, including prearm/postarm or multiarm comparison groups.

5. Study outcomes include viral suppression, retention and ART adherence.

6. Studies published in English language.

7. Studies from all countries, high-income, middleincome and low-income settings, published between 2010 and 2020.

The Population/Patient/Problem, Intervention, Comparison, Outcome and Time (PICOT)-based inclusion criteria has been summarised in table 1 . Studies that are not available in English language will be excluded, as well as review papers, editorials and letters.

The decision to only include studies reported in English language was necessitated by the limitations in capacity and resources among the review team, which restricts the review to English language only. Studies may include younger children below 10 years or young adults over 19 years, and justification for inclusion will be provided if these are included in the analysis. Additionally, other studies may focus on adherence interventions and viral load suppression for all people living with HIV, including adolescents in the study population or sample. Age disaggregation will be performed where there are age overlaps and the authors have provided age-disaggregated data.

\section{OUTCOME MEASURES}

Viral load suppression: although WHO viral load suppression threshold in 1000 copies $/ \mathrm{mL}$, more recent studies 


\begin{tabular}{|c|c|}
\hline $\begin{array}{l}\text { Patient } \\
\text { population }\end{array}$ & $\begin{array}{l}\text { Adolescents aged } 10-19 \text { years living with HIV, acquired perinatally or behaviourally, with challenges } \\
\text { suppressing their viral load }\end{array}$ \\
\hline Comparisons & $\begin{array}{l}\text { Studies may report comparisons on quantitative measures of retention, adherence and viral load suppression } \\
\text { between intervention and standard care (prerm/postarm or multiarm comparison groups) }\end{array}$ \\
\hline Outcomes & $\begin{array}{l}\text { Primary outcome-viral load suppression } \\
\text { Secondary outcomes - retention in care and ART adherence }\end{array}$ \\
\hline Time & 2010-2020 \\
\hline $\begin{array}{l}\text { Other } \\
\text { considerations }\end{array}$ & Language: English \\
\hline
\end{tabular}

Study designs: quantitative and mixed methods

Geographical area: global

ART, antiretroviral therapy.

may have used lower levels of viral load counts to define viral load suppression or disaggregate low level viraemia and suppression. All definitions of viral load suppression will be considered in this review. Where different cut-offs for viral load suppression are used, we will run sensitivity analyses.

ART adherence: adherence can be measured using pill counts, pharmacy refill data, self-reporting, as well as physician/nurse assessments and appointment keeping. Self-report is a common measure of adherence although there are concerns about its validity (limitation in correlation to viral loads), and socially desirable responses from especially younger individuals may overestimate adherence. ${ }^{27}$ More robust measures such as Medical Events Monitoring System and other new technologies will all be considered in this review. Optimal adherence will be considered to be at $\geq 95 \%$ of prescribed medication doses taken per specified period.

Retention in HIV care: approaches to evaluating retention in HIV care may be determined from missed clinic appointments or based on kept clinic visits. Retention variables may also include interruption in treatment, lost to follow-up, transferred-out or died. A subanalysis of the different categories of adolescents who are not still engaged in care will be performed if possible (when different retention variables are reported).

\section{SEARCH STRATEGY FOR IDENTIFICATION OF STUDIES}

The search strategy was developed with the assistance of the faculty librarian who will provide further guidance and advice in conducting the searches for the review. A comprehensive database search of literature reporting on treatment interventions and viral load suppression will be systematically conducted in six major databases: PubMed/ MEDLINE, Sabinet, EBSCOhost, CINAHL, Scopus and ScienceDirect. Grey literature searches will be performed on OpenGrey, Grey Literature report, International AIDS
Conference abstract archive, and HIV and Adolescence. We will search the identified databases for all articles published between 2010 and 2020 that meet the specified selection criteria. The search syntax will first be used to perform a database search on PubMed/MEDLINE to test and optimise the strategy, which will subsequently be replicated and adapted as needed for the other database searches. Full-text articles will be sourced using a list of key words and terms for the search strategy using the "AND" and "OR" Boolean operators as appropriate. The search terms will include "Adolescents", "antiretroviral therapy", "retention", "adherence", "viral load", "suppression", "unsuppressed". The search strategies used for the selected databases are provided in online supplemental material, titled "ALL Database search Results".

\section{STUDY SELECTION}

Studies will be included in this systematic review according to the PICOT mnemonics. We will also consider studies done using quantitative or mixed methods, with a worldwide search without any particular geographical location limitations. Restricting reviews to a particular study design, such as randomised controlled trial (RCTs) only, may restrict findings to effectiveness only rather than seeking answers to more complex phenomena such as why an intervention may or may not be effective. ${ }^{28}$

The selection, screening and reporting of this review will be done in accordance with the reporting guidance provided in the Preferred Reporting Items for Systematic Reviews and Meta-Analyses (PRISMA) Protocol 2015 guidelines. ${ }^{29}$ Selected studies will be collated and exported into Mendeley Reference Management Software, which will be used to manage the citations and recording the number of hits for each article from all databases, as well as removing duplicate records. ${ }^{30}$ Two reviewers will independently review titles and abstracts of all articles retrieved to assess eligibility for inclusion. 
The two reviewers will further review full texts of the selected articles independently, to establish compliance with the eligibility criteria for inclusion in the final assessment. ${ }^{26}$ Any disagreements on inclusion or exclusion of any article will be referred to a third reviewer authorised to resolve any disagreements for inclusion of articles. A PRISMA flow diagram will be presented to describe the study selection decisions made.

\section{DATA EXTRACTION AND QUALITY ASSESSMENT}

Conducting a quality assessment may determine the inclusion or exclusion of an initially selected study, and this should consider the robustness (validity and reliability) of findings of a particular study. ${ }^{28}$ We will create a data extraction matrix in Microsoft Excel to capture study characteristics and outcomes data, such as author(s), study year and duration, publication year, geographical location, target population, sample, purpose, study design, intervention, data collection and analyses, outcomes, results, limitations and conclusions. Only the data reported in the articles will be extracted and used in the quality assessment although additional information may be sought from authors for clarification.

\section{DATA ANALYSIS/SYNTHESIS: ASSESSMENT OF EVIDENCE USING THE GRADING OF RECOMMENDATIONS ASSESSMENT, DEVELOPMENT AND EVALUATION SYSTEM}

The two primary review authors will independently assess the selected articles using the Grading of Recommendations Assessment, Development and Evaluation considerations to downgrade or upgrade the certainty of evidence on the interventions and the specific outcomes reported by each study. Independent conclusions will be drawn on how the interventions compare to each other, on the specified treatment outcomes considering the estimates of effects, and the certainty of the evidence (risk of bias, confidence in evidence/consistency in effects, quality of evidence, imprecision, indirectness and publication bias). ${ }^{31}{ }^{32}$ The quality of evidence and risk of bias for non-RCTs will be assessed using the Critical Appraisal Skills Programme Checklist and the Risk of Bias in Nonrandomised Studies of Interventions (ROBINS-I) Assessment Tool, respectively. ${ }^{33}$ Studies which included clinical or medication issues such as drug resistance, and not necessarily adherence and retention challenges only, may be considered with appropriate justifications, especially reports on recent improved ART regimens.

We will conduct a meta-analysis only if the interventions, research questions or focus, and study participants allow for a meaningful meta-analysis. If possible, we will analyse results from the review by mode of infection among the adolescents included in meta-analysis. The meta-analysis will be conducted using Stata Statistical Software: Release V.16 (StataCorp). The $\chi^{2}$ test and the Higgins $\mathrm{I}^{2}$ statistic will be used to quantify statistical heterogeneity by estimating the variability between study estimates, with $\mathrm{I}^{2}$ statistic values of $25 \%, 50 \%$ and $75 \%$ representing low, medium and high heterogeneity, respectively. ${ }^{34}$ Forest plots will be used to visualise the extent of heterogeneity or homogeneity among the selected studies.

Publication bias described as the tendency by authors to report positive findings, will be presented and visualised on a funnel plot, as well as statistically assessed using the Harbord's regression test to assess the asymmetry of the funnel plot. ${ }^{35} 36$ The contour-enhanced funnel plot will be used to distinguish between asymmetry caused by publication bias versus other causes, which may not have been successfully accomplished by the standard method. ${ }^{37}$ If it is not possible to do a meta-analysis, the findings extracted onto the Excel spreadsheet described above will be summarised and reported in a table form presenting key components of the studies. Subgroup analysis may include facility versus community-based interventions and younger versus older adolescents.

\section{PATIENT AND PUBLIC INVOLVEMENT}

No patients or public population is involved in the development and conducting of this study, as this is a protocol for a systematic review of already available literature.

\section{ETHICS AND DISSEMINATION}

The systematic review entails abstracting and reviewing already publicly available data rather than any involvement of participants, therefore, no ethical clearance or approval will be required.

We plan to share results of this review with relevant policy-makers and guidelines developers, programme managers and service providers, particularly providing evidence-based guidance for interventions targeting unsuppressed adolescents. We also plan to publish the results of the review, as well as sharing the findings with key stakeholders using platforms such as conferences, and presentations through webinars and the HIV Project Extension for Community Health Outcomes. ${ }^{38}$

Acknowledgements Ms Karen Cook, Senior Librarian: Community and Health Sciences Branch Library, at the University of the Western Cape, for assisting with developing the search strategy and the data base searches.

Contributors FKM conceived the idea, developed the research questions and study methods and contributed meaningfully to the drafting and editing, he also approved the final manuscript. BvW aided in developing the research questions and study methods contributed meaningfully to the drafting and editing and approved the final manuscript.

Funding Self-funded.

Competing interests None declared.

Patient consent for publication Not required.

Provenance and peer review Not commissioned; externally peer reviewed.

Supplemental material This content has been supplied by the author(s). It has not been vetted by BMJ Publishing Group Limited (BMJ) and may not have been peer-reviewed. Any opinions or recommendations discussed are solely those of the author(s) and are not endorsed by BMJ. BMJ disclaims all liability and responsibility arising from any reliance placed on the content. Where the content includes any translated material, BMJ does not warrant the accuracy and reliability of the translations (including but not limited to local regulations, clinical guidelines, 
terminology, drug names and drug dosages), and is not responsible for any error and/or omissions arising from translation and adaptation or otherwise.

Open access This is an open access article distributed in accordance with the Creative Commons Attribution Non Commercial (CC BY-NC 4.0) license, which permits others to distribute, remix, adapt, build upon this work non-commercially, and license their derivative works on different terms, provided the original work is properly cited, appropriate credit is given, any changes made indicated, and the use is non-commercial. See: http://creativecommons.org/licenses/by-nc/4.0/.

ORCID iD

Farai Kevin Munyayi http://orcid.org/0000-0003-4410-7005

\section{REFERENCES}

1 Bekker L-G, Siberry GK, Hirnschall G. Ensuring Children and Adolescents Are Not Left Behind [Internet]. [cited 2020 May 6], 2018. Available: https://journals.Iww.com/jaids

2 UNICEF. HIV and AIDS in Adolescents - UNICEF Data [Internet]. [cited 2020 Dec 11], 2020. Available: https://data.unicef.org/topic/ adolescents/hiv-aids/

3 Sherr L, Cluver LD, Toska E, et al. Differing psychological vulnerabilities among behaviourally and perinatally HIV infected adolescents in South Africa - implications for targeted health service provision. AIDS Care 2018;30:92-101.

4 UNICEF. Adolescent HIV prevention - UNICEF DATA [Internet]. [cited 2020 Sep 10], 2020. Available: https://data.unicef.org/topic/hivaids/ adolescents-young-people/

5 UNAIDS. UNAIDS data 2019 [Internet]. [cited 2020 May 6], 2019. Available: https://www.unaids.org/sites/default/files/media_asset/ 2019-UNAIDS-data_en.pdf

6 WHO. WHO | Definition of key terms. WHO [Internet]. [cited 2016 Oct 11], 2013. Available: http://www.who.int/hiv/pub/guidelines/arv2013/ intro/keyterms/en/

7 UNAIDS. Ending the AIDS epidemic for adolescents, with adolescents: a practical guide to meaningfully engage adolescents in the AIDS response. Geneva, 2016.

8 UNAIDS. Global HIV \& AIDS statistics - 2020 fact sheet | UNAIDS [Internet]. [cited 2020 Dec 12], 2020. Available: https://www.unaids. org/en/resources/fact-sheet

9 Ssali L, Kalibala S, Birungi J. Retention of adolescents living with HIV in care, treatment, and support programs in Uganda. [cited 2018 Feb 27], 2014. Available: http://www.hivcore.org/Pubs/Uganda AdolHAART_Rprt.pdf

10 Grimsrud AT, Pike C, Bekker LG. The power of peers and community in the continuum of HIV care [Internet]. Vol. 8, The Lancet Global Health. Elsevier Ltd. 2020 [cited 2020 Nov 16]. p. e167-8. Available: https://pubmed.ncbi.nlm.nih.gov/31924538/

$11 \mathrm{Kim}$ S-H, Gerver SM, Fidler S. Adherence to antiretroviral therapy in adolescents living with HIV: systematic review and meta-analysis. AIDS 2014;28:1945-56.

12 Nachega JB, Mills EJ, Schechter M. Antiretroviral therapy adherence and retention in care in middle-income and low-income countries: current status of knowledge and research priorities. Curr Opin HIV AIDS 2010;5:70-7.

13 Ridgeway K, Dulli LS, Murray KR, et al. Interventions to improve antiretroviral therapy adherence among adolescents in low- and middle-income countries: a systematic review of the literature. PLoS One 2018;13:e0189770-33.

14 MacPherson P, Munthali C, Ferguson J, et al. Service delivery interventions to improve adolescents' linkage, retention and adherence to antiretroviral therapy and HIV care. Trop Med Int Health 2015;20:1015-32.

15 WHO. WHO | Viral suppression after 12 months of antiretroviral therapy in low- and middle-income countries: a systematic review. WHO [Internet]. cited 2017 Jul 13, 2013. Available: http://www.who. int/bulletin/volumes/91/5/12-112946/en/

16 WHO. WHO | Viral suppression for HIV treatment success and prevention of sexual transmission of HIV [Internet]. [cited $2020 \mathrm{Dec}$ 12], 2018. Available: https://www.who.int/hiv/mediacentre/news/ viral-supression-hiv-transmission/en/

17 Ridgeway K, Dulli LS, Murray KR, et al. Interventions to improve antiretroviral therapy adherence among adolescents in low- and middle-income countries: a systematic review of the literature. PLoS One 2018;13:e0189770.

18 Ammon N, Mason S, Corkery JM. Factors impacting antiretroviral therapy adherence among human immunodeficiency virus-positive adolescents in sub-Saharan Africa: a systematic review. Public Health 2018;157:20-31.

19 Hudelson C, Cluver L. Factors associated with adherence to antiretroviral therapy among adolescents living with HIV/AIDS in low- and middle-income countries: a systematic review. AIDS Care 2015;27:805-16.

20 Koss CA, Hosek SG, Bacchetti P, et al. Comparison of measures of adherence to human immunodeficiency virus preexposure prophylaxis among adolescent and young men who have sex with men in the United States. Clinical Infectious Diseases 2018;66:213-9.

21 Casale M, Carlqvist A, Cluver L. Recent interventions to improve retention in HIV care and adherence to antiretroviral treatment among adolescents and youth: a systematic review. AIDS Patient Care STDS 2019;33:237-52.

22 Reif LK, Abrams EJ, Arpadi S. Interventions to Improve Antiretroviral Therapy Adherence Among Adolescents and Youth in Low- and Middle-Income Countries: A Systematic Review 2015-2019 [Internet]. Vol. 1, AIDS and Behavior. Springer; 2020 [cited 2020 Jul 31]. p. 1. Available: /pmc/articles/PMC7223708/?report=abstract

23 Mavhu W, Willis N, Mufuka J, et al. Effect of a differentiated service delivery model on virological failure in adolescents with HIV in Zimbabwe (Zvandiri): a cluster-randomised controlled trial. Lancet Glob Health 2020;8:e264-75.

24 Diress G, Dagne S, Alemnew B, et al. Viral load suppression after enhanced adherence counseling and its predictors among high viral load HIV seropositive people in North Wollo zone public hospitals, northeast Ethiopia, 2019: retrospective cohort study. AIDS Res Treat 2020;2020:1-9.

25 Nasuuna E, Kigozi J, Babirye L, et al. Low HIV viral suppression rates following the intensive adherence counseling (IAC) program for children and adolescents with viral failure in public health facilities in Uganda. BMC Public Health 2018;18:1048.

26 Egger M, Smith GD. Principles of and procedures for systematic reviews. in: systematic reviews in health care: meta-analysis in context. 2nd edn. Wiley Blackwell, 2008: 23-42.

27 Müller AD, Jaspan HB, Myer L, et al. Standard measures are inadequate to monitor pediatric adherence in a resource-limited setting. AIDS Behav 2011;15:422-31.

28 Grant MJ, Booth A. A typology of reviews: An analysis of 14 review types and associatedmethodologies [Internet]. Vol. 26, Health Information and Libraries Journal. John Wiley\& Sons, Ltd; 2009 [cited 2020 Nov 3]. p. 91-108. Available: https://onlinelibrary.wiley.com/doi/ full/

29 Moher D, Shamseer L, Clarke M, et al. Preferred reporting items for systematic review and meta-analysis protocols (PRISMA-P) 2015 statement. Syst Rev 2015;4.

30 Price A, Price A. Mendeley and More for Systematic Reviews. Int Netw Knowl about Wellbeing [Internet]. 2016 Apr 26 [cited 2020 Nov 3]. Available: http://www.ithinkwell.org/mendeley-and-more-forsystematic-reviews/

31 Mearns H, Otiku PK, Shelton M. Screening strategies for adults with type 2 diabetes mellitus: A systematic review protocol [Internet]. Vol. 9, Systematic Reviews. BioMed Central; 2020 [cited 2020 Nov 16]. p. 156. Available: https://systematicreviewsjournal.biomedcentral.com/ articles/10.1186/s13643-020-01417-3

32 Brignardello-Petersen R, Izcovich A, Rochwerg B, et al. Grade approach to drawing conclusions from a network meta-analysis using a partially contextualised framework. BMJ 2020;371:m3907.

33 Haghighat R, Steinert J, Cluver L. The effects of decentralising antiretroviral therapy care delivery on health outcomes for adolescents and young adults in low- and middle-income countries: a systematic review. Glob Health Action 2019;12:1668596.

34 Mengesha MM, Ajema D, Teshome A, et al. The association between diagnosis disclosure and adherence to antiretroviral therapy among adolescents living with HIV in sub-Saharan Africa: A protocol for systematic review and meta-analysis [Internet]. Vol. 9, Systematic Reviews. BioMed Central; 2020 [cited 2020 Nov 7]. p. 160. Available: https://systematicreviewsjournal.biomedcentral.com/articles/10. 1186/s13643-020-01420-8

35 Egger M, Smith GD, Schneider M, et al. Bias in meta-analysis detected by a simple, graphical test. BMJ 1997;315:629-34.

36 Rao G, Lopez-Jimenez F, Boyd J, et al. Methodological standards for meta-analyses and qualitative systematic reviews of cardiac prevention and treatment studies: a scientific statement from the American Heart Association. Circulation 2017;136:e172-94.

37 Chaimani A, Mavridis D, Salanti G. A hands-on practical tutorial on performing meta-analysis with Stata. Evid Based Ment Health 2014;17:111-6.

38 Bikinesi L, O’Bryan G, Roscoe C, et al. Implementation and evaluation of a project echo telementoring program for the Namibian HIV workforce. Hum Resour Health 2020;18:1-10. 